\title{
Service-Related Factors Associated With Newborn Care Practices by Mothers in Mutare, Zimbabwe: A Cross-Sectional Study
}

\author{
Edhina Chiwawa ${ }^{1}$, Maxwell Mhlanga ${ }^{2}$, Professor Auxillia Munodawafa ${ }^{1} \&$ Fadzayi Mutseyekwa $^{1}$ \\ ${ }^{1}$ The College of Health, Agriculture and Natural Sciences, Africa University, Mutare, Zimbabwe \\ ${ }^{2}$ College of Health Sciences, University of Zimbabwe, Mutare, Zimbabwe \\ ${ }^{2}$ Correspondence: Maxwell Mhlanga, College of Health Sciences, University of Zimbabwe, P.O Box A178 \\ Avondale Harare. E-mail: profmaxmhlanga7@gmail.com
}

Received: February 13, 2020 Accepted: March 13, 2020 Online Published: April 7, 2020

doi:10.5539/gjhs.v12n6p37

URL: https://doi.org/10.5539/gjhs.v12n6p37

\begin{abstract}
Neonatal mortality remains very high in Zimbabwe (29/1,000 live births). Service-related factors have been linked to the care-giver knowledge and newborn care practices. In Manicaland Province, care-seeking behaviors for fever (the main symptom for childhood infections) and knowledge levels of neonatal danger signs remains unacceptably lower than the national averages. A cross-sectional descriptive and analytic design was carried out to determine service-related factors to newborn care practices and their association with neonatal outcomes in Mutare District. Purposive sampling was used to select health facilities and systematic random sampling was used to select participants. A structured interviewer administered questionnaire was used to collect data which was then entered and analyzed using Epi-Info version 7.2. Descriptive and multivariate analysis were performed on data collected from 349 participants. Practice of the five assessed recommended newborn care practices was high. Exclusive breastfeeding $(62 \%)$, early initiation of breastfeeding $(74 \%)$, good cord care $(73 \%)$, care-seeking for neonatal illness within $24 \mathrm{hrs}$ of onset of fever (82\%) and keeping baby warm was at $58 \%$. Statistically significant independent factors associated with the recommended newborn care practices were post-natal stay for more than 72 hours at the clinic $(\mathrm{AOR}=0.56 ; 95 \% \mathrm{CI}: 0.12-0.87, \mathrm{p}=0.000)$, and delivery at the health facility $(\mathrm{AOR}=0.43$; 95\%CI: 0.21-0.77; $\mathrm{p}=0.000$ ). Service delivery had a substantial influence on newborn care practices in Mutare district and influenced neonatal outcomes. Improving newborn care outcomes requires both effective service delivery and community health system strengthening to promote good newborn care.
\end{abstract}

Keywords: neonatal health, service-related factors, care-practices

\section{Introduction}

Globally, significant progress and gains have been noted in child survival. The decline in neonatal mortality has been very slow between 1990 and 2015 compared to that of post-neonatal under-5 mortality, with $99 \%$ of maternal and newborn mortality occurring in the developing world (WHO, 2016). About 1.16 million African babies die in the first 28 days of life and the highest burden of neonatal mortality is among marginalised and poorest populations in Sub-Saharan Africa (Tura et al., 2014). These deaths are attributed to conditions of labour, intrapartum and poor immediate newborn care practices (Warren et al., 2015).

Essential Newborn Care (ENC) is a pool of measures required by each newborn baby regardless of size or place of birth and these are meant to protect the newborn from adverse environmental conditions (Coutin, 2015). Components of newborn care (which encompass breastfeeding, cord care, eye care, thermoregulation, management of asphyxia, recognition of danger signs, immunization and care of the low birth weight infant) and newborn resuscitation have been proven to reduce incidence of neonatal mortality rate and still birth rate (Malhotra et al., 2014). Inequities to access and utilisation of material, neonatal and child health services have been associated with health facility related factors such as the quality of service rendered and the distance to the nearest health facility especially in rural areas and peri-urban areas, leading to low prevalence of essential newborn care practices (Karim et al., 2015; Tegene et al., 2015 and Berhane et al., 2014).

In Zimbabwe, the Multiple Indicator Cluster Survey, (MICS, 2014) indicates that the neonatal mortality levels have been on the increase across the successive five-year periods. The neonatal mortality rate was 20 deaths per 1,000 live births between 2000 and 2004; 25 deaths per 1,000 live births between 2005 and 2009; and lastly 29 
deaths per 1,000 live births between 2010 and 2014. Manicaland Provinces is among the top two populated provinces in the country, coming second after Harare Metropolitan province. Although an improvement has been noted across all areas in the performance of maternal, newborn and child health indicators in the MICS (2014) compared to the 2010/11 Zimbabwe Demographic Health Survey performance in Manicaland Province, the achievements are still below the average national coverage with regards to key indicators necessary for neonatal survival. The rates for institutional delivery, skilled attendance at delivery, post-partum stay in health facility for 3 days or more coverage $(72 \%, 72.2 \%$, and $25.5 \%)$ remain still low compared to the national coverages of $(79.6 \%$, $80 \%$ and $32.1 \%$ ) respectively (MICS, 2014).

The health and survival of the neonate largely depends on the quality of care both at health facility and in the community available during pregnancy, delivery and post-partum period including the care given to the neonate by the mother or care giver at home. Since 2010 a lot of effort has been put by the Ministry of Health and Child Care (MOHCC) across all the pillars of the health delivery system: Human resources for health, Health financing, Governance and leadership which include coordination and policy setting, Health service delivery, Information surveillance and research and Access to essential drugs and supplies to try and improve newborn outcomes.

The ability to identify that the newborn has a danger sign and the health seeking behaviour for neonatal illness by mothers highly relies on the mother knowledge about neonatal danger signs. In the MICS study, very few mothers/care givers of children under-5 years were able to recognize fast or difficult breathing as signs for seeking care immediately. Below is a table indicating the knowledge level of women aged 15-49 years who are mothers or primary caregivers of children under age 5 by symptoms that would cause them to take a child under age 5 immediately to a health facility, and percentage of mothers who recognize fast or difficult breathing as signs for seeking care immediately in Manicaland compared to the national coverage (Zimbabwe MICS, 2014).

The quality of care both at health facility and in the community available during pregnancy, delivery and postpartum period has much impact on immediate recognition of danger signs in the neonate during birth and postpartum period as a result, it is necessary for the mother and family to understand these aspects of childbirth and newborn care and be prepared to react to the potential dangers signs such as hypothermia, jaundice, unable to feed, apnoea, bleeding through the umbilicus, convulsions, and fever (World Vision International, 2016). These low levels of interaction between health care providers and mothers of the newborn during the most critical time of a newborn baby`s life calls for attention in view of the fact that more than half of the newborn deaths occur in the first seven days of life in the care of the mothers and primary care givers at home. This study sought to identify service-related factors and determine their association with newborn care practices and neonatal health outcomes and make improvements in neonatal care.

This study hypothesises that health service provision factors are associated with uptake of recommended newborn practices and neonatal outcomes.

\section{Methods}

\subsection{Study Setting and Design}

An analytical cross-sectional study was conducted among women of child bearing age who delivered a live baby or attended PNC at selected health facilities in Mutare District of Manicaland Province. The study was carried out in catchment areas of six MNH high volume health facilities of Mutare District in Manicaland Province. Mutare district comprises Mutare Urban and Rural. Rural health facilities that were selected based on 2015 District Health Information System 2 (DHIS 2) included: Marange Rural Hospital, Zimunya Clinic and Odzi Clinic. Urban health facilities were Chikanga, Sakubva Poly Clinic and Dangamvura Poly Clinic. The primary study population comprised women of child bearing age who delivered a live baby or attended Post-Natal-Care (PNC) clinics in the 3 months preceding the data collection period at the selected health facilities. The study was conducted between May 2016 and May 2017.

\subsection{Sampling and Sampling Procedure}

Manicaland province is among the top two malaria high burdened provinces in Zimbabwe. Considering that fever is the most prevalent symptom for children under the age of five, the study used care seeking for fever as a proxy measure for 'general care seeking for childhood including neonatal illness' and for 'knowledge of newborn danger signs'. The prevalence for care seeking during fever in children under the age of 5 years in Manicaland province is 51 percent (MICs, 2014). Taking 0.05 as the margin of error at $95 \%$ Confidence Interval (CI) to calculate the sample size, a total of 382 participants will be interviewed. The calculation is as shown below:

Formula used: $n=\left(z^{\wedge} 2 p q\right) / d^{\wedge} 2$ 


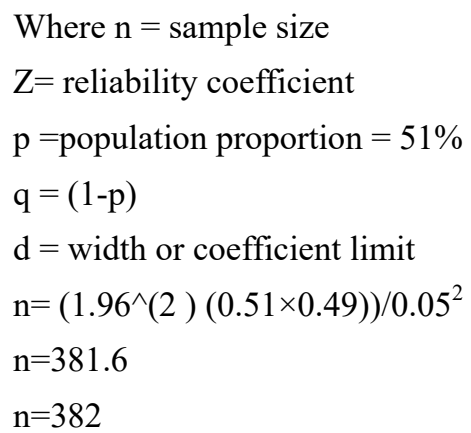

Assuming a non-response rate of $10 \%$, the sample size will be 421 participants

Six primary health facilities were purposively selected, 3 from the urban community and 3 from the rural community of Mutare District in Manicaland Province. Selection of the facilities was based on the District Health Information System 2 (DHIS 2) reported volume of maternal and newborn health services that were offered in 2015. Referral centres like Sakubva District Hospital and Mutare Provincial Hospital were excluded from the study. Statistics for postnatal care at 6weeks during the 2015-year period were used as an indicator to select the high-volume sites. Rural health facilities that were selected included: Marange Rural Hospital, Zimunya Clinic and Odzi Clinic. Urban health facilities were Chikanga, Sakubva Poly Clinic and Dangamvura Poly Clinic.

All women of child bearing age who delivered a live baby or attended Post-Natal-Care clinics at the selected health facilities during the three-month period preceding the data collection were included in the sampling frame and approached for enrolment into the study. The study had a calculated sample of 421 participants. Two stage sampling method was used. The first stage was purposive sampling of health facilities based on the 2015 DHIS2 reported maternal and newborn health statistics (delivery and postnatal care data reported in 2015) and the second stage was the systematic random sampling of the participants at each health facility. Six high volume sites were selected, 3 from the Mutare City Health (Chikanga, Sakubva and Dangamvura Health facilities) and 3 from the Mutare Rural District namely Marange, Odzi and Zimunya health facilities. Population sample was proportionally distributed between the six selected health facilities.

The second stage comprised the selection of the primary participants. Listing of all women delivered or who attended PNC clinics in the three months preceding the data collection at the selected clinics was done to develop sampling frames for the different selected clinics. Systematic random sampling method was used to select the participants with no replacement. The first subject was randomly selected from the total population. Based on the required sample, a sampling interval was determined using the formula $k=\frac{\mathrm{N}}{\mathrm{n}}$ where $k$, is the sampling interval (skip interval), $\mathrm{N}$ is the total population in the sampling frame for a selected health facility and $\mathrm{n}$ is the required sample size for each health facility.

\subsection{Data Collection and Analysis}

A structured questionnaire was used for data collection. This was administered in the form of face to face interviews. Information was collected on the following independent variables:

1. Health service factors-distance from the nearest health facility, ANC attendance, place of delivery for the last birth, use of PNC services during previous births, Health education sessions attended, home visits by VHWs during pregnancy or post-natal period, participation in mothers support groups (IYCF, Mbereko or community $\mathrm{MNCH}$ care groups)

The outcome variables for newborn care practices were, care seeking for neonatal illness with $24 \mathrm{hrs}$ of onset of fever, keeping baby warm, good cord care explained as keeping the cord stump dry and not applying anything on the cord stump, early initiation of breastfeeding explained as initiating breast feeding within the first hour after delivery, exclusive breast feeding explained as giving breast milk only to the baby for the first six months after birth and bathing the baby after 48 hours.

Pretesting of the data collection instruments was done at Fen valley and Rowa health facilities (Mutare city and Mutare rural respectively). These health facilities have similar characteristics with the selected health facilities. The Data collection tool was shared with content experts and their feedback was considered to modify the tool as necessary. Data was collected at each of the selected health facility independently. Face to face interviews were conducted at the health facilities using a structured questionnaire. At the end of each data collection day, the questionnaires were checked for accuracy and consistency. Questionnaires with inaccurate and inconsistent information were rejected and completed questionnaires were kept under lock and key to protect respondent 
confidentiality.

Data was collected, entered into a data base and analysed using Epi-Info version 7.2 Descriptive analysis was done in terms of frequency, proportions and mean. Bivariate analysis was performed to assess associations of each of the independent variables with the explanatory variables. Multiple logistic regression models were developed for multivariate analysis to identify independent factors that were associated with good neonatal care and knowledge of newborn danger signs.

\subsection{Ethical Considerations}

Consent to carry out the study was sought from Africa University, Manicaland Provincial Medical Director and the District Medical Officer of Mutare district. Ethical approval was sought from the Africa University Research Ethics Committee. Participants were asked to provide written informed consent before the interview. All adolescent mothers under the age of 18 years are considered to be young mothers so consented on their own. Confidentiality of information was maintained throughout the study. Names and other identifying information were no required from the participant and questionnaires were coded. Participation was voluntary and respondents were allowed to withdraw from the study at any time to maintain privacy, interviews were conducted individually respecting the respondent's right to privacy.

\section{Results and Analysis}

\subsection{Response Rate}

The response rate was $83 \%$ (349 participants).

\subsection{Factors Associated With Newborn Care Practices Among Women of Child Bearing Age}

Bivariate analysis was carried out on each of the newborn care practices, crude odds ratios (COR) and their corresponding $95 \%$ confidence intervals $(\mathrm{CI})$ as well as p-values were presented. The results are presented in tables below.

Table 1a. Health-care service-related factors and their association with neonatal care practices and outcomes

\begin{tabular}{|c|c|c|c|c|}
\hline \multirow[b]{2}{*}{ Variable } & \multicolumn{2}{|c|}{ Early initiation of breast feeding } & \multirow[b]{2}{*}{ OR $(95 \% \mathrm{CI})$} & \multirow[b]{2}{*}{ p-value } \\
\hline & $\begin{array}{l}\text { Yes } \\
\text { n (\%) }\end{array}$ & $\begin{array}{l}\text { No } \\
\text { n(\%) }\end{array}$ & & \\
\hline \multicolumn{5}{|c|}{ Received ANC services } \\
\hline Yes & $250(73.7)$ & $40(11.8)$ & \multirow{2}{*}{$32.03(13.22-80.35)$} & \multirow{2}{*}{$0.003^{*}$} \\
\hline No & $8(2.4)$ & $41(12.1)$ & & \\
\hline \multicolumn{5}{|c|}{ Received health education on breast feeding } \\
\hline Yes & $235(78.6)$ & $37(12.4)$ & & \\
\hline No & $19(6.3)$ & $8(2.7)$ & $2.67(0.99-7.06)$ & 0.993 \\
\hline \multicolumn{5}{|l|}{ Place of delivery } \\
\hline Health facility & $244(73.5)$ & $26(7.8)$ & \multirow{2}{*}{43.51 (19.09-101.5) } & \multirow{2}{*}{$0.001 *$} \\
\hline Home & $11(3.3)$ & $51(15.4)$ & & \\
\hline \multirow{3}{*}{ Variable } & \multicolumn{2}{|c|}{ Good cord care } & \multirow{3}{*}{ OR (95\% CI) } & \multirow{3}{*}{ p-value } \\
\hline & Yes & No & & \\
\hline & n (\%) & $\mathbf{n}(\%)$ & & \\
\hline \multicolumn{5}{|c|}{ Post-natal stay in clinic } \\
\hline Less than $72 \mathrm{hrs}$ & $120(58.3)$ & $21(10.2)$ & \multirow{2}{*}{$0.37(0.10-1.22)$} & \multirow{2}{*}{0.120} \\
\hline $72 \mathrm{hrs}$ and above & $61(29.6)$ & $4(1.9)$ & & \\
\hline \multicolumn{5}{|c|}{ Post-natal death in the family } \\
\hline Yes & $12(31.6)$ & $12(31.6)$ & \multirow{2}{*}{$1.80(0.38-8.71)$} & \multirow{2}{*}{0.178} \\
\hline No & $5(13.1)$ & $9(23.7)$ & & \\
\hline
\end{tabular}




\begin{tabular}{|c|c|c|c|c|}
\hline \multicolumn{5}{|c|}{ Being assisted with newborn care } \\
\hline Yes & $100(39.2)$ & $59(23.1)$ & \multirow{2}{*}{$0.93(0.11-7.93)$} & \multirow{2}{*}{0.686} \\
\hline No & $90(35.3)$ & $6(2.4)$ & & \\
\hline \multicolumn{5}{|c|}{ Post-natal stay in clinic } \\
\hline Less than $72 \mathrm{hrs}$ & $120(58.3)$ & $21(10.2)$ & \multirow{2}{*}{$0.37(0.10-1.22)$} & \multirow{2}{*}{0.120} \\
\hline $72 \mathrm{hrs}$ and above & $61(29.6)$ & $4(1.9)$ & & \\
\hline
\end{tabular}

*- represents statistical significance.

Women who delivered at a facility were 43.59 times more likely to initiate breast feeding early within one hour after delivery as compared with those who delivered at home and the results were statistically significant $(\mathrm{COR}=$ 43.51; 95\%CI: $19.09-101.50 ; \mathrm{p}=0.001)$. Having received Antenatal care services was significantly associated with early initiation of breast feeding the baby. Those who received Antenatal care services were 23.03 times more likely to initiate breast feeding early as compared those who did not receive Antenatal care services $(\mathrm{COR}=23.03$ : 95\% CI: $13.22-80.35, \mathrm{p}=0.003^{*}$. Again, those who delivered at the facility were 49.22 times more likely to practice good cord care as compared to those who delivered at home ( $\mathrm{COR}=49.22 ; 95 \% \mathrm{CI}$ : $19.46-128.47, \mathrm{p}=0.00)$.

Table 1b. Health-care service-related factors and their association with neonatal care practices and outcomes

\begin{tabular}{|c|c|c|c|c|}
\hline \multirow{3}{*}{ Variable } & \multicolumn{2}{|c|}{$\begin{array}{l}\text { Care seeking for neonatal illness } \\
\text { within } 24 \text { hrs of onset of fever }\end{array}$} & \multirow{3}{*}{ OR $(95 \%$ CI $)$} & \multirow{3}{*}{ p-value } \\
\hline & & No & & \\
\hline & $\mathrm{n}(\%)$ & $\mathrm{n}(\%)$ & & \\
\hline \multicolumn{5}{|c|}{ Distance from nearest health facility } \\
\hline Less than $10 \mathrm{~km}$ & $276(83.4)$ & $43(13.0)$ & \multirow{2}{*}{$0.58(0.33-0.87)$} & \multirow{2}{*}{$0.015^{*}$} \\
\hline Greater than $10 \mathrm{~km}$ & $11(3.3)$ & $1(0.3)$ & & \\
\hline \multicolumn{5}{|c|}{ Knowledge of newborn danger signs } \\
\hline Yes & $55(14.6)$ & $16(4.2)$ & \multirow{2}{*}{$0.4(0.06-0.89)$} & \multirow{2}{*}{$0.006^{*}$} \\
\hline \multirow[t]{2}{*}{ No } & $276(72.9)$ & $32(8.3)$ & & \\
\hline & \multicolumn{2}{|c|}{ Exclusive breast feeding } & \multirow{3}{*}{ OR $(95 \%$ CI $)$} & \multirow{3}{*}{ p-value } \\
\hline \multirow[t]{2}{*}{ Variable } & Yes & No & & \\
\hline & n (\%) & n(\%) & & \\
\hline \multicolumn{5}{|c|}{ VHW PNC home visits } \\
\hline Yes & $28(8.4)$ & $59(17.8)$ & \multirow{2}{*}{$0.63(0.38-1.06)$} & \multirow{2}{*}{0.052} \\
\hline No & $105(31.6)$ & $14041.2)$ & & \\
\hline \multicolumn{5}{|c|}{ Received health education after delivery } \\
\hline Yes & $5(2.3)$ & $2(0.9)$ & \multirow{2}{*}{$0.84(0.14-6.44)$} & \multirow{2}{*}{0.564} \\
\hline No & $161(72.5)$ & $54(23.4)$ & & \\
\hline
\end{tabular}

*- represents statistical significance. VHW means Village Health Workers.

Distance from nearest HF, and knowledge of danger signs were also some of independent variables that had an influence on care seeking for neonatal illness within $24 \mathrm{hrs}$ of onset of fever.(COR:0.58;95\% Cl:0.33-0.87; $\mathrm{p}=0.015$; and COR: $0.4 ; 95 \% \mathrm{Cl}: 0.06-0.89 ; \mathrm{p}=0.006)$ respectively. Those who had knowledge of danger signs were 0.4 times more likely to seek care for neonatal illness within $24 \mathrm{hrs}$ of onset of fever.

3.3 Factors associated with keeping the baby warm soon after delivery.

In this study, the variable of interest was the time the mothers took before giving the baby the first bath after delivery. At least $59 \%$ of the participants interviewed bathed their babies 48 hours after delivery. 


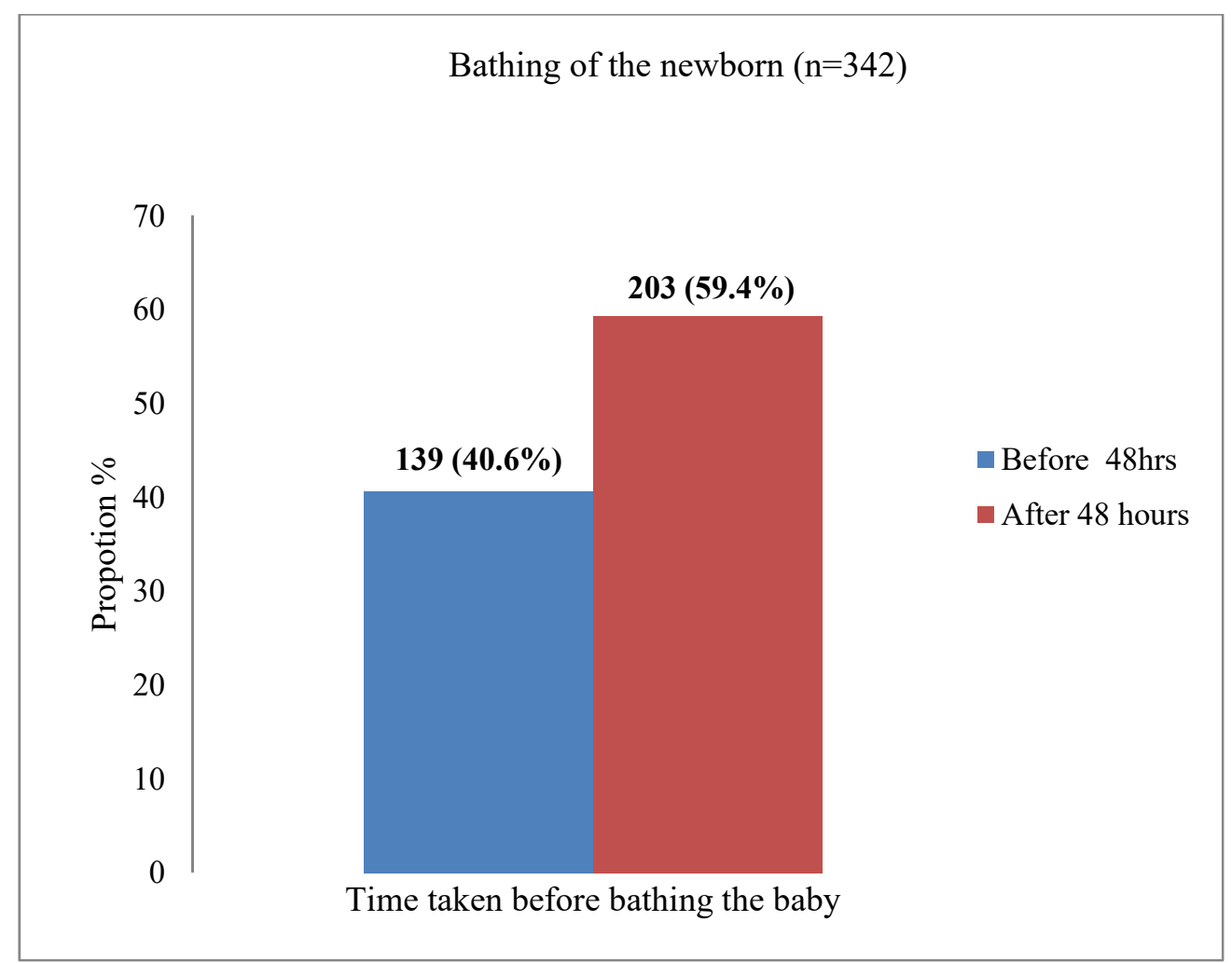

Figure 1. Bathed the Baby within 48 Hrs and After 48 Hrs after Delivery (N=342)

Bivariate analysis was also carried out to identify the factors associated with bathing baby after 48 hours after birth. Postnatal stay at health facility were not statistically associated with the recommended practice of bathing the baby after 48 hours after birth.

\subsection{Independent factors associated with newborn care practices}

Multivariate analysis was done to allow for efficient estimation of measures of association between independent factors and newborn care practices while controlling for a number of confounding variables simultaneously. Stepwise logistic regression analysis is the technique that was used in multivariate analysis. Adjusted Odds Ratios (AOR) and their corresponding p-values and 95\% confidence intervals (CI) were calculated. Table 3 below shows final results of stepwise logistic regression analysis.

Table 2. Independent Factors Associated With Newborn Care Practices

\begin{tabular}{llll}
\hline Factor & AOR & $\mathbf{9 5 \%}$ CI & p-value \\
\hline Received health education during ANC visits & 0.07 & $0.01-1.28$ & 0.141 \\
Post-natal stay for more than 72 hours at clinic & $\mathbf{0 . 5 6}$ & $\mathbf{0 . 1 2}-\mathbf{0 . 8 7}$ & $\mathbf{0 . 0 0 0 *}$ \\
Delivery at health facility & $\mathbf{0 . 4 3}$ & $\mathbf{0 . 2 1}-\mathbf{0 . 7 7}$ & $\mathbf{0 . 0 0 0 *}$ \\
Received health education on breast feeding & 0.12 & $0.01-2.31$ & 0.465 \\
Having a post-natal death in the family & 0.78 & $0.25-3.66$ & 0.762 \\
Knowledge of newborn danger signs & 0.79 & $0.33-1.67$ & 0.537 \\
\hline
\end{tabular}

*- statistically significant variable.

Results displayed in Table 2 shows that statistically significant independent factors associated with the recommended newborn care practices were post-natal stay for more than 72 hours at the clinic (AOR $=0.56 ; 95 \% \mathrm{CI}$ : $0.12-0.87, \mathrm{p}=0.000$ ), and delivery at the health facility ( $\mathrm{AOR}=0.43 ; 95 \% \mathrm{CI}: 0.21-0.77 ; \mathrm{p}=0.000$ ). 


\section{Discussion}

Provision of antenatal and post -natal care by a skilled attendant have been identified as key facility based clinical care strategies to deliver some of the cause specific effective interventions for the prevention of newborn deaths and poor community mobilization and health promotion activities have resulted in poor maternal and child health outcomes (Kabwijamu et al., 2016). The individualized counseling sessions during ANC and PNC provide important opportunities for the health care providers to disseminate key messages on neonatal danger signs and newborn care to the mother or care giver to ensure preparedness, informed decision making and appropriate prompt action to seek health care for the newborn illnesses. This will also reduce the prevalence of numerous unscientific and unhygienic practices and social taboos in neonatal care which makes newborns extremely vulnerable (Yaregal et al., 2019). The ability of mothers to select appropriate feeding practices is significantly influenced by the individualized counseling support that is provided through formal health services especially during antenatal care.

The results of this study revealed that, access and utilization of safe motherhood preparatory practices for newborn care was very high with attendance of at least four ANC visits (85\%), institutional delivery $77.4 \%$ and $90 \%$ of the women received health education on breastfeeding. Delivering in the health facility was taken as a proxy indicator for having access to a skilled birth attendant during delivery. Newborn care promotive interventions like postnatal stay in the health facility for at least 72 hours and PNC home visits by Village Health Workers were below 30\%, less than $10 \%$ of the women received health education during postnatal care and belonged to a maternal and newborn health support group. This result clearly point to a weak health system at both community and facility level. Poor community mobilization structures keep care-givers disempowerment in maternal and child care resulting in the perpetuation of harmful newborn care practices such as applying potential harmful substances to the cord stump, early bathing and giving prelacteal feeds (Kabwijamu et al., 2016).

PNC services be it in the health facility or provided in the community are meant to reinforce and provide a supportive environment for uptake of and adherence to good newborn care practices, low coverage of these services may mean that even if women have the knowledge about what is to be done, because of lack of reinforcement may not feel empowered enough to comply with the recommended newborn care practices. Effective targeting of women during pregnancy through special ANC days and follow up mechanisms after delivery could improve the knowledge and newborn care practices post-delivery. It is estimated that about $75 \%$ of neonatal deaths can be avoided by simple low cost tools such as use of sterile blades to cut the umbilical cord, using clean dry clothes to wrap and keep babies warm and early initiation of breastfeeding (Kumola, 2015).

The results of this study also reveal that attending at least 4 ANC visits (OR: 32.03; Cl 95\% 13.22-80.35; $\mathrm{p}=0.003$ ) was associated with early initiation of breast feeding with women who had attended at least four ANC visits being 32.03 times more likely to initiate breast feeding within the first hour after delivery than those who had less number of visits of who had not attended ANC. Consistent with this finding is that timing of first ANC visit was noted to be a predictor of good newborn care practices with women who initiated ANC early in the first trimester being 2 times more likely to give good neonatal feeding to their babies (AOR:2.26;95\%Cl1.43-3.57; $<0.001$ ) than women who initiated their first ANC late (Kayom et al., 2015).Contradicting to this finding, Ayiasi et al (2014) noted in their study that adherence to four or more ANC visits did not show any statistically significant relationship with the recommended or desired newborn care practices.

Having delivered at the health facility ( AOR: $0.43 ; 95 \% \mathrm{Cl}: 0.21-0.77 ; \mathrm{p}=0.000$ ) and having post-natal stay for more than 72 hours at the clinic ( AOR: $0.56 ; 95 \% \mathrm{Cl}: 0.12-0.87 ; \mathrm{p}=0.000$ ), are factors which were noted in this study to be associated with the newborn care practices among women of child bearing age in Mutare district. However, having an institutional delivery was neither associated with the good practices of delaying the first bath nor the recommended exclusive breast-feeding practice. This result differed with what was reported in North East Ethiopia where number of ANC visits and mode of delivery were factors associated with newborn care practices among postnatal mothers (Yaregal et al., 2019). Home deliveries were associated with bathing of newborns after 24 hours of birth.

Generally, there is consensus that access to skilled birth attendants during ANC, delivery and PNC has many benefits for the mother baby -pair. Consistent with the delivered in the health facility reported good cord care practices compared to $63 \%$ of those who delivered at home and were 1.2 times more likely to initiate breast feeding within the first hour after delivery when compared to those who delivered at home (Kayom et al., 2015). Ayiasi et al (2014) in their study reported that women who delivered in the health facility ( $A O R=3.72$ : $p<0.001)$ were more likely to report complete thermal care for the newborn.

Attending the recommended number of ANC visits and use of skilled birth attendant at delivery was noted to be 
significantly associated with increased knowledge of newborn danger signs and was protective against neonatal mortality in Latin America (Munos et al., 2014). Skilled birth attendant during postnatal period in a health facility is a crucial element of the continuum of care approach for the mother and the newborn. Skilled attendant at every birth influences the care that is provided to the newborn and maternal knowledge of neonatal danger signs at home. According to Saaka and Iddrisu (2014), use of a skilled birth attendant was associated with receipt of positive feeding practices: facility born babies were slightly more likely to be breast fed within $24 \mathrm{hrs}$ relative to those delivered at home. This window of opportunity should be harnessed by health workers using a supermarket approach to comprehensively empower mother post-delivery on how to take care of themselves and the newborn to prevent complications and infections.

Postnatal care is care given to the woman and baby following child birth up to six weeks. The mother and the baby are expected to stay under supervision at a health facility up to 72 hours after delivery. Use of postnatal care is very important for identification and prompt treatment of complications. During postpartum period, physical, social and mental problems can emerge thus; postnatal care includes both preventive and curative services. During this time mothers also receive health education on child care, diet, breastfeeding, weaning and family planning. Like during $\mathrm{ANC}$, the role of the skilled attendant during PNC is to reinforce information on essential newborn care, hygiene, family planning, nutrition, care of the newborn at home and danger signs to watch for when at home (Khanal, Adhikari, Karkee, and Gavidia, 2014).

With regards to health promotion intervention such as health education, postnatal home visits by community-based health workers and support groups, some studies have revealed the positive association between provision of health education through individualised or group counselling and newborn care practices (Memon, Khan, Soofi, Baig, and Bhutta 2015). Of note is the intervention study that was done in Northern Pakistan in which community based health workers were trained to implement a newborn health preventive package which included capacitating community based health workers to be able to do postnatal home visits, provide health education and counselling to the mothers in relation to newborn care and provide referrals for newborn illness. The results of the intervention revealed that reported practices of colostrum administration as first feed $(83 \%$ vs $64 \%, \mathrm{p}<.001)$, initiation of breast feeding within one hour after birth $(55 \%$ vs $40 \%, \mathrm{p}<.001)$ and EPI vaccination for newborns was comparatively higher in intervention areas than in control areas. Women in intervention areas were less likely to report practice of traditional cord application $(51 \%$ vs $71 \%, \mathrm{p}<.001)$ than the women in control areas (Memon et al., 2015).

This is contrary to the findings of this study which revealed that although $290(85 \%)$ of the participants received health education during ANC, 7 (3.1\%) received health education after delivery, 87 (26.2\%) received a Village Health Worker postnatal home visits and14 (4.3\%) of the participants belonged to an MNH support group, there was no association between these factors and some of the newborn care practices such as early care seeking for fever related illness in the first 24 hours of the onset of fever, good cord care and delayed bathing to keep the baby warm.

The low coverage $(4.3 \%)$ of women who reported that they have received health education after delivery and the lack of association between receiving health education and the good newborn care practices in this study can be an indication that health workers during this time concentrate more on offering clinically oriented services at the expense of the less technically involving services like giving health education. A study carried out in Kenya showed that provision of clinically inclined services like provision of palpation and vaccination during ANC were over $90 \%$ while provision of health education during same ANC was 14.4\% (Van Eijk, Hanneke, Odhiambo, Ayisi, Blockland, Rosen, Adazu, Slutsker, \& Lindablade, 2006).

Nikiema, Beninguisse and Haggerty, (2009) in their multi country study in Sub Saharan Africa noted that slightly less than half of the participants indicated that they had not received any educational information on danger signs in pregnancy which resulted in delays in care seeking in case of complications. In support of the above, Kayon et al., (2015) also noted that although $90 \%$ of their participants reported attending ANC, only $45 \%$ received teachings on appropriate cord care.

\section{Conclusion}

The study demonstrated that service delivery factors have a substantial influence on newborn care practices by mothers in Mutare district. In this study, access to ANC services was significantly associated with early initiation of breastfeeding rates and place of delivery. Community mobilization to improve newborn care practices through effective health promotion activities that reach saturation coverage at household level to improve maternal knowledge on newborn care practices and care seeking behavior. 
Independent health-facility related factors associated with the recommended newborn care practices were having a post-natal stay for more than 72 hours at the clinic, delivery at the health facility and having received health education on breastfeeding. The governments in low to medium income countries should focus on strengthening the capacity of the primary health care centres in rural areas to retain women for 72 hours post-delivery. This practice offers health professionals a window of opportunity to teach, demonstrate and observe newborn care practices. This will go a long way in reducing neonatal morbidity and mortality.

\section{Competing Interests Statement}

The authors declare that there are no competing or potential conflicts of interest.

\section{References}

Abera, M., Nega, A., Kedir, T., Shiyate, S., \& Agegrehu, B. (2018). Essential newborn care practice and its predictors among mother who delivered within the past six months in Chencha District, Southern Ethiopia. PLoS One, 13(12). https://doi.org/10.1371/journal.pone.0208984

Amolo, L., Irimu, G., \& Njai, D. (2017). Knowledge of postnatal mothers on essential newborn care practices at the Kenyatta National Hospital: a cross sectional study. Pan Afr Med J., $28,97$. https://doi.org/10.11604/pamj.2017.28.97.13785

Ayiasi, R., M, Criel, B., Orach, C. G., Nabiwemba, E., \& Kolsteren, E. (2014). Primary healthcare worker knowledge related to prenatal and immediate newborn care: a cross sectional study in Masindi, Uganda. BMC Health Services Research, 14, 65. https://doi.org/10.1186/1472-6963-14-65

Ayiasi, R. M., Kasasa, S., Criel, B., Orach, C. G., Nabiwemba, E., \& Kolsteren, E. (2014). Is antenatal care preparing mothers to care for their newborns? A community-based cross-sectional study among lactating women in Masindi, Uganda. BMC Pregnancy and child birth, 14, 114. https://doi.org/10.1186/1471-2393-14-114

Berhanu, D., \& Avan, B. I. (2014). Community Based Newborn Care: Baseline report summary, Ethiopia. IDEAS, London School of Hygiene \& Tropical Medicine: London.

Coutin, A. (2015).Essential Obestritic and Newborn Care: Practical guide for midwives, doctors with obstetrics training and health care personnel who deal with obstetrics emergencies. ed. 2015.

Karim, A.M., Tamire, A., Medhanyie, A. A., \& Betemariam, W. (2015). Changes in equity of maternal, newborn, and child health care practices in 115 districts of rural Ethiopia: implications for the health extension program. BMC Pregnancy Childbirth, 15, 238. https://doi.org/10.1186/s12884-015-0668-z

Kayom, V. O., Kakuru, A., \& Kiguli, S. (2015). Newborn Care Practices among Mother-Infant Dyads in Urban Uganda. International Journal of Pediatrics, 2015. http://dx.doi.org/10.1155/2015/815938

Kumola, A. M. (2015). Newborn care practices among postnatal mothers in Garissa county, Kenya. 2015.

Kabwijamu, L., Waiswa, P., Kawooya, V., Nalwadda, C.K., Okuga, M., \& Nabiwemba, E. L. (2016). Newborn Care Practices among Adolescent Mothers in Hoima District, Western Uganda. PLoS One, 11(11), e0166405. https://doi.org/10.1371/journal.pone.0166405

Malhotra, S., Zodpey, S. P., Vidyasagaran, A. L., Sharma, K., Raj, S. S, \& Neogi, S. B. (2016). Assessment of essential newborn care services in secondary-level facilities from two districts of India. Journal of health, population, and nutrition, 32(1), 130-41.

Memon, Z. A., Khan, G. N., Soofi, S. B., Baig, I. Y., \& Bhutta, Z. A. (2015). Impact of a community-based perinatal and newborn preventive care package on perinatal and neonatal mortality in a remote mountainous district in Northern Pakistan. BMC Pregnancy and Childbirth, 15, 106. https://doi.org/10.1186/s12884-015-0538-8

Misgna, G. H., Gebru, B. H., \& Birhanu, M. M. (2016). Knowledge, practice and associated factors of essential newborn care at home among mothers in Gulomekada District, Eastern Tigray, Ethiopia. BMC Pregnancy and Childbirth. https://doi.org/10.1186/s12884-016-0931-y

Nalwadda, C. K., Waiswa, P., Guwatudde, D., Kerber, K., Peterson, S., \& Kiguli, J. (2014). 's long as the umbilical cord gets off, the child ceases to be called a newborn: sociocultural beliefs and newborn referral in rural Uganda. Glob Health Action, 724386. https://doi.org/10.3402/gha.v8.24386

Owor, M. O., Matovu, J. K. B., Murokora, D., Wanyenze, R. K., \& Waiswa, P. (2016). Factors associated with adoption of beneficial newborn care practices in rural Eastern Uganda: a cross-sectional study. $B M C$ 
Pregnancy Childbirth,16, 83. https://doi.org/10.1186/s12884-016-0874-3

Reshma, Z. K., \& Sujatha, R. (2014). Cultural practices and beliefs on newborn care among mothers in a selected hospital of Mangalore Taluk. Nitte University Journal of Health Science: NUJHS, 4(2), 2249-7110.

Tegene T., Andargie G., Nega A., \& Yimam, K. (2015). Newborn Care Practice and Associated Factors among Mothers who gave Birth within One Year in Mandura District, Northwest Ethiopia. Clinics in Mother and Child Health, 2015. https://doi.org/10.4172/2090-7214.1000172

Tura, G., Mesganaw, F., \& Alemayehu, W. (2014). Determinants and Causes of Neonatal Mortality in Jimma Zone, Southwest Ethiopia: A Multilevel Analysis of Prospective Follow Up Study. PLOS ONE, 9(9).

Warren, C., Daly, P., Toure, L., \& Mongi, P. (2015). Opportunities for Africa's Newborns: Postnatal care. 2015.

WHO. To reduce child mortality. (2016; http://www.who.int/mediacentre/factsheets/fs178/en/.

Workineh, Y., Hailu, D., Gultie, T., Degefu, N., Mihrete, M., Shimele, M., Mahino, M., Guesh, M., \& Alemu. M. (2014). Knowledge of Obstetric Danger Signs and its Associated Factors in Arba Minch Town, Ethiopia. American Journal of Health Research, 2(5), 255-259. dhttps://doi.org/10.11648/j.ajhr.20140205.16

WVI. Essential Newborn Care. (2016). http://www.wvi.org/health/intervention-2-essential-newborn-care

Yaregal, S., Meaza, E., Alemayehu, T., Desalegn, A., \& Tsegaye, G. (2019). Newborn care practices and its determinants among postnatal mothers in Dessie Referral Hospital, Northeast Ethiopia. BMC Res Notes, 12, 96. https://doi.org/10.1186/s13104-019-4133-3

Zimbabwe National Statistics Agency (ZIMSTAT). (2014). Multiple Indicator Cluster Survey, Final Report. Harare, Zimbabwe.

\section{Copyrights}

Copyright for this article is retained by the author(s), with first publication rights granted to the journal.

This is an open-access article distributed under the terms and conditions of the Creative Commons Attribution license (http://creativecommons.org/licenses/by/4.0/). 